\title{
Enhanced Recovery Pathway for Right and Left Colectomy: Comparison of Functional Recovery
}

\author{
Anne Kummer ${ }^{1} \cdot$ Juliette Slieker $^{1} \cdot$ Fabian Grass $^{1} \cdot$ Dieter Hahnloser $^{1}$ \\ Nicolas Demartines ${ }^{1} \cdot$ Martin Hübner $^{1}$
}

Published online: 18 May 2016

(C) Société Internationale de Chirurgie 2016

\begin{abstract}
Background Enhanced recovery (ERAS) guidelines do not differentiate between left- and right-sided colectomies, but differences in recovery have been reported for the two procedure types. We aimed to compare compliance with the ERAS protocol and outcomes after right versus left colectomy.

Methods Between June 2011 and September 2014, all patients undergoing elective colonic resection were treated according to a standardized ERAS protocol and entered a prospective database. This retrospective analysis compared right and left colectomy regarding application of the ERAS pathway, bowel recovery, complications, and hospital stay.

Results Eighty-five patients with right colectomy matched well with 138 left-sided resections for baseline demographics. Overall compliance with the ERAS protocol was $76 \%$ for right versus $77 \%$ for left colectomy patients $(p=0.492)$. First flatus occurred at postoperative day 2 in both groups $(p=0.057)$; first stool was observed after a median of 3 (right) and 2 days (left), respectively $(p=0.189)$. Twenty patients $(24 \%)$ needed postoperative nasogastric tube after right colectomy compared to 11 patients $(8 \%)$ after left colectomy $(p=0.002)$. Overall complication rates were 49 and $37 \%$ for right and left colectomy, respectively $(p=0.071)$. Median postoperative length of stay was 6 days (IQR 4-9) after right and 5 days (IQR 4-7.5) after left colectomy $(p=0.020)$.

Conclusion Overall compliance with the protocol was equally high in both groups showing that ERAS protocol was applicable for right and left colectomy. Functional recovery however, tended to be slower after right colectomy, and postoperative ileus rate was significantly higher. More cautious early feeding after right colectomy should be considered.
\end{abstract}

Meeting presentations: Congrès Suisse, Société Suisse de Chirurgie, Berne, 20-22nd May 2015.

Trial registration number: Research Registry UIN 372.

Electronic supplementary material The online version of this article (doi:10.1007/s00268-016-3563-5) contains supplementary material, which is available to authorized users.

Nicolas Demartines

demartines@chuv.ch

1 Department of Visceral Surgery, University Hospital of Lausanne (CHUV), Rue du Bugnon 46, 1011 Lausanne, Switzerland

\begin{abstract}
Abbreviations
ERAS Enhanced recovery after surgery

POD Postoperative day
\end{abstract}

\section{Introduction}

Perioperative care has significantly evolved during the last decade for major abdominal surgery, especially colorectal surgery. Multimodal approaches with the use of enhanced recovery protocols allowed to reduce postoperative complications, length of stay, and costs [1-5]. The enhanced 
recovery after surgery (ERAS) guidelines provides a summary of evidence-based measures in elective colonic surgery [6]. These measures aim for a decrease in surgical stress, maintenance of physiologic functions, and early mobilization, along with avoidance of prophylactic abdominal drain, routine nasogastric tube, and adoption of a stringent intravenous fluid administration policy [6]. The relationship between application of the enhanced recovery protocol (=compliance) and improvement in clinical outcome has been reproducibly demonstrated [3, 7]. The same protocol is advocated for left- and right-sided resections [6].

Outcomes after colonic resection were widely reviewed but few studies have focused on differences in outcome after right versus left colectomy. These studies evaluated complications, length of stay, and mortality [8-12]. They reported a higher rate of anastomotic leak [8, 10] and reoperations for sepsis [9], more superficial surgical site infections [9, 11], and more intraoperative complications $[12,13]$ after left colectomy. Conversely, one study showed more postoperative complications after right colectomy, such as urinary tract infection, pneumonia, and ileus [13]. Length of stay tended to be longer after rightsided resection in one study [9], but shorter in another [11]. Mortality was similar in both groups [8, 9, 11, 13, 14].

There is a common belief that recovery after right colectomy is more problematic and longer, although the surgical act is regarded less challenging and faster as compared to left-sided resections. However, data about functional recovery confirming or refuting this perception are lacking. It remains, therefore, unknown whether one single pathway fits equally for left- and right-sided colectomies.

The objective of this study was to compare right and left colectomy with regard to compliance with the ERAS protocol, functional outcomes, and clinical results.

\section{Methods}

\section{Study design}

This is a single-center, retrospective cohort study based on a prospective database. The institutional ethics committee approved the study (no. 46/15).

The study was conducted in accordance with the STROBE criteria (http://strobe-statement.org/) and registered under www.researchregistry.com (UIN 372).

\section{Patients}

In May 2011, a standardized enhanced recovery protocol for elective colonic resection was implemented at the
University Hospital of Lausanne and applied systematically [4]. Since then, demographic and surgical information, application of protocol items, and functional and clinical outcomes until 30 days postoperatively were recorded routinely into a prospective database, the ERAS Interactive Audit System.

All patients undergoing elective colon resection were treated from June 2011 according to the ERAS protocol, and no patient was excluded from the pathway; consecutive patients until September 2014 were included in the present analysis. Patients undergoing right colectomy or ileocaecal resection were referred to the right colectomy group and patients undergoing left colectomy or sigmoidectomy were included in the left colectomy group.

\section{Surgical procedures}

All surgeries were performed or supervised by a boardcertified surgeon. Our institution has a strong mission for surgical education and hence a high turnover of junior staff surgeons. Overall, 13 different surgeons performed the colon resections of the study cohort, but always with a staff surgeon. In fact, surgical technique was standardized and the staff surgeon always present, so there were very few differences among junior surgeons. Laparoscopic resection was favored whenever thought to be feasible by the senior surgeon. End-to-end stapled anastomosis was performed after left colectomy [15, 16] and ileocolic side-to-side stapled anastomosis for right colectomy [17].

\section{Enhanced recovery after surgery protocol}

The institutional enhanced recovery protocol was based on the published ERAS guidelines [6] and used in recent institutional publications [4, 18]. This protocol includes 21 items (online resource).

\section{Outcome measures}

Adherence to the ERAS $^{\circledR}$ protocol was assessed as dichotomous variable for every item of the institutional enhanced recovery protocol. Percentages for each individual item were calculated as compliant patients divided by total patients for both groups. Overall compliance was calculated as number of fulfilled items divided by 21 , the total number of items. The adherence to the protocol was separately calculated for the pre-, intra-, and postoperative phase using the number of items in each phase, respectively, as denominator.

Functional outcomes included time to first passage of stool and flatus and time to tolerate solid food, measured in number of postoperative days (POD). Level of pain was assessed by use of a visual analogical scale (0-10 points) 
and by the time to pain control with oral analgesics, measured in number of POD. Postoperative ileus was defined as the necessity of insertion of a nasogastric tube; indication for nasogastric tube placement was based on the following criteria: abdominal distension, no bowel movements, and nausea/vomiting occurring later than $24 \mathrm{~h}$ after surgery. These criteria are in line with a recent consensus definition of Vather et al. [19].

Clinical outcomes included complication rate and length of stay. Postoperative complications occurring within 30 postoperative days were graded according to the Clavien classification [20] on a 5-point severity scale. Grade III to IVb complications were defined as severe complications. For patients with more than one complication, the most severe complication was considered. Length of stay was measured from day of surgery until day of hospital discharge.

\section{Subgroup analysis}

A significant higher rate of postoperative ileus was observed in the right colectomy group, with a trend for slower functional recovery. As open surgical approach represents a risk factor for delayed bowel recovery [21-24] and this approach was more often used for right colonic resection, it was decided to perform a post hoc analysis including only patients who underwent completed laparoscopic surgery.

\section{Statistical analysis}

Descriptive statistics are reported as median (interquartile range) or mean ( \pm standard deviation) as appropriate for continuous variables and absolute or relative frequencies for categorical variables. Continuous variables were compared using the Mann-Whitney $U$ test or Student's $t$ test. Categorical variables were compared with Chi-squared or Fisher's exact test. All tests were two-sided. A $p$ value of less than 0.05 was considered significant.

Univariate risk factors for insertion of nasogastric tube and potential confounders were entered into a logistic multivariate regression model. Only variables with a $p$ value of less than 0.1 on univariate testing were included.

Data were analyzed by use of IBM $^{\circledR}$ SPSS $^{\circledR}$ statistics 22.0 (IBM, Armonk, NY, USA).

\section{Results}

\section{Demographics and surgical characteristics (Table 1)}

Between June 2011 and September 2014, 223 consecutive and unselected patients underwent elective colonic resection and were included in our prospective ERAS database.
Eighty-five patients underwent right colectomy or ileocaecal resection and 138 patients underwent left colectomy or sigmoidectomy, respectively. One hundred and fourteen men and 109 women, with a mean age of $64( \pm 16)$ years were treated. Both groups were similar with regard to demographic data, besides a higher BMI in the left colectomy group. Malignant disease was more common for patients with right-sided colectomy (69\%) compared with patients with left-sided resections $(52 \%)(p=0.012)$. There was a trend to choose laparoscopic approach more often for left $(76 \%)$ than for right $(66 \%)$ colonic resection $(p=0.124)$, with a conversion rate of 13 versus $9 \%$ $(p=0.521)$. The added number of primarily open and converted procedures was 29/85 for right versus 33/138 for left colectomy ( $p=0.124)$.

One patient received a loop ileostomy after right-sided resection and two patients after left colectomy. Three colostomies were performed in the left group.

\section{Compliance with the enhanced recovery protocol}

Overall compliance with the enhanced recovery protocol was similar in both groups (76\% in right and $77 \%$ in leftsided resections, $p=0.492$ ). Compliance with the items of the preoperative phase was 97 and $98 \%$ for right and left colon resection groups, respectively $(p=0.522)$, while intraoperative phase showed a compliance of 78 and $70 \%$, respectively $(p=0.005)$. Postoperative items were followed with $65 \%$ compliance in right versus $68 \%$ in left colectomy groups $(p=0.152)$. The application of individual items is detailed in Fig. 1.

\section{Functional outcomes}

First flatus occurred in both groups after a median of two postoperative days (IQR 1-3 and 1-2 for right and left colectomy groups, respectively, $p=0.057$ ). First passage of stool occurred on POD 3 (IQR 2-4) after right colectomy and on POD 2 (IQR 2-3) after left colectomy $(p=0.189)$. Time to tolerate solid food was 3 POD (IQR 1-3) in patients with right and 2 POD (IQR 2-3) in patients with left colectomy $(p=0.638)$. Postoperative ileus occurred in 20 patients $(24 \%)$ with right colectomy and in 11 patients $(8 \%)$ with left colectomy $(p=0.002)$.

Pain control with oral analgesics was achieved on POD 2 for both groups (IQR 1-3 for right versus 1-2 for left colectomy groups, respectively, $p=0.008$ ). Level of pain was similar in both groups (Fig. 2).

\section{Complications and length of stay (Table 2)}

Overall, 42 patients with a right colectomy (49\%) and 51 patients with a left colectomy (37\%) developed 
Table 1 Demographics and operation characteristics of patients undergoing right versus left colectomy

\begin{tabular}{llll}
\hline & Right colectomy $(N=85)$ & Left colectomy $(N=138)$ & $p$ value \\
\hline Age (years) & $64.4 \pm 19.2$ & $63.4 \pm 14.3$ & $0.675^{\mathrm{a}}$ \\
Gender ratio (male:female) & $45: 40$ & $69: 69$ & $0.682^{\mathrm{b}}$ \\
Body mass index $\left(\mathrm{kg} / \mathrm{m}^{2}\right)$ & $25.0 \pm 4.9$ & $26.8 \pm 5.1$ & \\
ASA grade & & $106(77)$ & $0.012^{\mathrm{a}}$ \\
I-II & $62(73)$ & $32(23)$ & \\
III-IV & $23(27)$ & $66(48)$ \\
Pathology & $26(31)$ & $72(52)$ \\
Benign & $59(69)$ & & \\
Malign & & $105(76)$ \\
Surgical approach & $56(66)$ & $15(11)$ \\
Laparoscopic & $21(25)$ & $18(13)$ \\
Open & $8(9)$ & $5(4)$ & $0.012^{\mathrm{b}}$ \\
Converted & $1(1)$ & $204 \pm 69$ \\
Stoma formation & $182 \pm 95$ & $10(7)$ & $0.124^{\mathrm{b}}$ \\
Surgery duration (min) & $10(12)$ & $0.008^{\mathrm{b}}$ \\
Intraoperative blood loss $\geq 500 \mathrm{ml}$ & & $0.521^{\mathrm{b}}$ \\
\hline
\end{tabular}

Values are presented as mean $\pm \mathrm{SD}$ or $N(\%)$ unless indicated otherwise

ASA American Society of Anesthesiologists, $S D$ standard deviation

a $t$ test

b Fisher's exact test

postoperative complications $(p=0.071)$. Severe complications occurred in $10(12 \%)$ patients in the right and 10 (7\%) patients in the left colectomy group $(p=0.104)$.

Three deaths occurred, all after left-sided resections. Causes were septic shock following anastomotic leakage, myocardial infarction with cardiac arrest, and hepatic failure due to Budd-Chiari syndrome.

Median postoperative length of stay was 6 days (IQR 4-9) in the right and 5 days (IQR 4-7.5) in the left colectomy group $(p=0.020)$. Postoperative stay in patients without complications was 4 days (IQR 3-5) in both groups $(p=0.252)$, while length of stay in patients with complications was 9.5 days (IQR 7-16) for right and 9 days (IQR 6-12.5) for left-sided resection $(p=0.343)$.

\section{Subgroup analysis including completed laparoscopic resections}

Fifty-six patients in the right colectomy group were compared to 105 patients in the left-sided resection group. Postoperative ileus rate was still significantly higher following laparoscopic right-sided resection, affecting 10 patients (18\%), compared to 4 patients (4\%) after laparoscopic left colectomy $(p=0.006)$. However, first passage of flatus occurred on POD 2 (IQR 1-2) in both groups, as well as first passage of stool (IQR 2-3). Time to tolerate solid food was 3 POD (IQR 1-3) in patients with right and 2 POD (IQR 2-3) in patients with left colectomy $(p=0.513)$.

\section{Risk factors for postoperative ileus: multivariate analysis}

As shown in Table 3, four factors were significantly associated with the occurrence of postoperative ileus in our study cohort. After multivariate analysis, only right colectomy was retained as independent risk factor for insertion of nasogastric tube.

\section{Discussion}

This study comparing functional recovery between rightand left-sided colectomies suggests a slower functional recovery and a significantly higher ileus rate after right colectomies. Overall compliance with the ERAS pathway was equally high among the two groups. Therefore, a more cautious early postoperative feeding protocol after right colectomy is suggested.

In this study, the observed postoperative ileus rate after right-sided resections was $24 \%$. As postoperative ileus is considered a grade II complication according to Clavien 
Fig. 1 Compliance with ERAS protocol by item in patients undergoing left compared to right colectomy

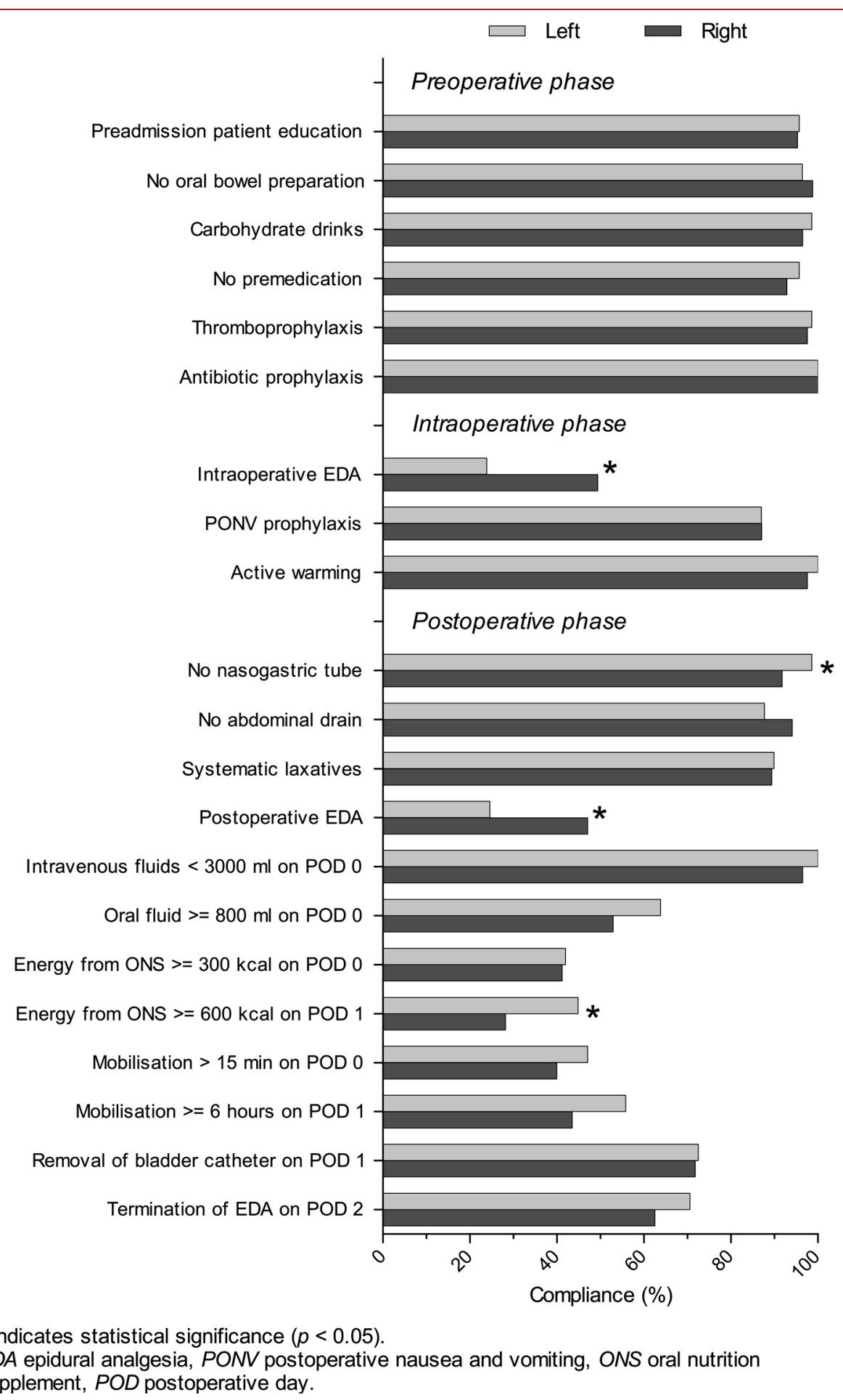

EDA epidural analgesia, PONV postoperative nausea and vomiting, ONS oral nutrition supplement, $P O D$ postoperative day. classification, it represents the majority of grade II complication in the present study cohort and hence accounts for the overall increased complication rates after right colectomy, even with laparoscopy. Overall, we observed an ileus rate after right- and left-sided resections of $13.9 \%$. This rate is similar to the findings of other publications $[21,25$,
26]. One study showed a higher ileus rate after right colectomies (38\%) compared to left-sided resections (14\%) in univariate analysis [22]. However, the authors provide no explanation for this finding. Furthermore, patients were not treated within a standardized ERAS pathway containing an array of preventive measures. 


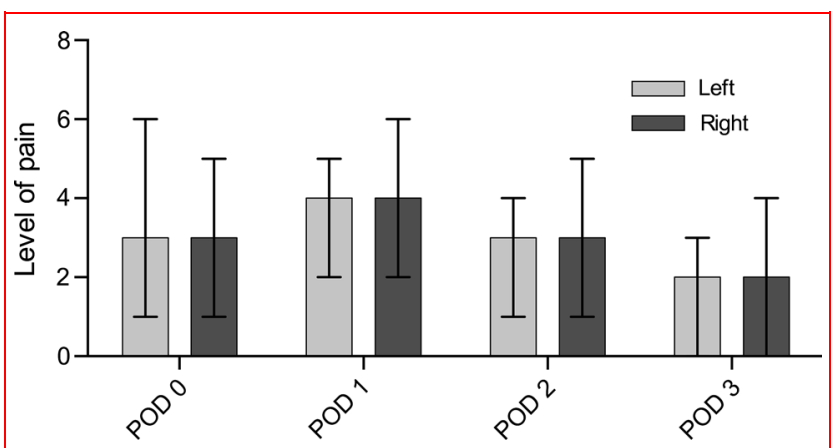

Fig. 2 Level of pain in patients undergoing left compared to right colectomy assessed with a visual analogic scale ( $0-10$ points)

The definition of postoperative ileus is inconsistent in the literature rendering comparison between studies problematic. We used therefore the objective endpoint of insertion of nasogastric tube as definition for the purpose of this study. Vather et al. have recently proposed a consensus statement $[19,27]$. The proposed definitions for postoperative ileus correspond to the clinical indications for nasogastric tube placement used in our study. Furthermore, this pragmatic approach has also been proposed in the recent systematic review of Wolthuis et al. [28] concluding «that reinsertion of the NG tube should be adopted as the standardized definition in the future».

In our cohort, demographics and surgical details were overall well matched. However, differences existed concerning several potential risk factors for delayed bowel recovery. These were in particular a higher BMI [26, 27, 29] and longer operative time [21, 25, 30], disfavoring leftsided resections. Several studies [21-24] have demonstrated lower ileus rates after a laparoscopic approach compared to open procedures. In our cohort, the rate of laparoscopic procedures was slightly lower in the rightsided resection group. However, the added number of primarily open and converted procedures was not significantly different, and might therefore not explain the higher incidence of ileus after right colectomy. Furthermore, the subgroup analysis including only laparoscopic cases confirmed a higher rate of ileus following right-sided resection. Finally, our hypothesis was strengthened by multivariate analysis retaining right colectomy as the only independent risk factor for postoperative ileus.

Vather et al. [27] identified several other factors associated with postoperative ileus following colorectal surgery, namely copious administration of intravenous crystalloids in the perioperative period and delayed mobilization. In our cohort, exactly the same perioperative protocol was applied for both comparative groups as recommended [6] and we did not identify any difference with regard to these important factors in our patients.

A recent publication [21] identified ileocolic anastomosis as a risk factor for postoperative ileus, compared to colocolic or colorectal anastomosis. The authors suggest that anastomosis with small bowel could play a role in ileus occurrence. This could be a hypothesis regarding our findings, but paucity of the literature on this topic limits further explanation.

The impact of stoma formation on the incidence of postoperative ileus is controversial in the literature. Intuitively, patients with an ostomy might have earlier signs of bowel motion (e.g., first flatus, stool) as an ostomy removes (parts of) colonic recovery from the equation. On the other hand, Millan et al. [31] identified ileostomy as a risk factor for postoperative ileus, while Thoker et al. [32] found no

Table 2 Clinical outcomes of patients undergoing right versus left colectomy

\begin{tabular}{llll}
\hline & Right colectomy $(N=85)$ & Left colectomy $(N=138)$ & $p$ value \\
\hline $\begin{array}{l}\text { Patients with complication(s) within 30 days } \\
\text { Complications grade }\end{array}$ & $42(49)$ & & \\
Grade I & $10(12)$ & $19(14)$ & $0.071^{\mathrm{a}}$ \\
Grade II & $22(26)$ & $19(14)$ & $0.839^{\mathrm{a}}$ \\
Grade III & $5(6)$ & $2(6)$ & $0.032^{\mathrm{a}}$ \\
Grade IV & $5(6)$ & $3(2)$ & $1.000^{\mathrm{a}}$ \\
Grade V & 0 & $5(4-7.5)$ & $0.109^{\mathrm{a}}$ \\
Postoperative stay (days) & $6(4-9)$ & $3(2)$ & $0.289^{\mathrm{a}}$ \\
Readmission within 30 days & $4(5)$ & $7(5)$ & $0.020^{\mathrm{b}}$ \\
Reoperation(s) & $6(7)$ & $0.432^{\mathrm{a}}$ \\
\hline
\end{tabular}

Values are presented as median (interquartile range) or $\mathrm{N}(\%)$

a Fisher's exact test

b Mann-Whitney $U$ test 
Table 3 Risk factors for reinsertion of nasogastric tube

\begin{tabular}{llr}
\hline & Univariate OR (95 \% CI) & Multivariate OR (95 \% CI) \\
\hline BMI $>25$ & $0.83(0.39-1.77)$ & - \\
Open or converted approach & $\mathbf{3 . 9 7}(1.81-8.67)$ & $2.21(0.90-5.42)$ \\
Right colectomy & $\mathbf{3 . 5 5}(1.61-7.86)$ & $\mathbf{3 . 3 2}(1.39-7.95)$ \\
Operative duration $>180 \mathrm{~min}$ & $\mathbf{2 . 3 3}(1.04-5.21)$ & $2.22(0.89-5.50)$ \\
Epidural analgesia & $\mathbf{3 . 9 4}(1.79-8.67)$ & $2.12(0.87-5.14)$ \\
IV fluid on day of surgery $>3000 \mathrm{ml}$ & $1.00(0.99-1.01)$ & - \\
\hline
\end{tabular}

Bold indicates statistical significance

$O R$ odds ratio, $C I$ confidence interval, $B M I$ body mass index, $I V$ intravenous

difference between patients with or without ostomy after rectal resection. In our study, removal of ostomy patients from the analysis did not change the results.

The overall compliance with the ERAS protocol in our analysis was similar to the rate observed in the literature [7, $18,33,34]$ and was equally high in both groups. Therefore, unequal application of the ERAS protocol does not explain the differences in functional outcomes. Detailed analysis of compliance with the intended ERAS protocol showed significant differences between the two groups for three items only; we found a higher rate of epidurals and prophylactic nasogastric tubes in the right colectomy group but a lower tolerance of oral supplements on POD 1. In line with the findings of Nelson et al. [35], our results confirm that prophylactic nasogastric drainage-nasogastric tube inserted in operating room and left in place in the postoperative phase-does not prevent ileus. The use of epidurals for colorectal surgery remains controversial, especially for laparoscopic resections. Concerning the open approach, bowel recovery tended to be faster with epidurals in some studies, while others did not find any difference [36]. Moreover, most of the included studies did not provide ERAS care to the included patients. For minimalinvasive resections within ERAS pathways, most recent studies could not find a benefit for epidurals that even appeared to slow down the recovery process in some studies [37, 38]. This is in accordance with the findings of the present study.

There is a panel of different strategies that could help to prevent ileus; a revision of some parts of the ERAS protocol by adding several of these new measures should be considered for right-sided resection. Some strategies could be implemented in the ERAS protocol in the light of recent therapies, as summarized in the review of Bragg et al. [39]. The systematic use of alvimopan, a peripheral opioid antagonist, allowed faster bowel function recovery and reduces length of stay [40-43]. Systematic lidocaine has shown to reduce time to bowel movement after abdominal surgery [44]. The stimulation of gut motility with chewing gum [45] or coffee [46] should be encouraged. Laxatives such as bisacodyl act on colon peristaltism and may confer additional benefit [47].

The present study has several limitations. Certain differences between the comparative groups exist and might have influenced outcomes. More open surgeries and more epidurals might have disfavored the right colectomy group. On the other hand, higher BMI was more prevalent in the group of left-sided resections. In addition, operative time trended to be longer for left-sided resections. After all, the present study aimed to compare non-selected right- versus left-sided resections. As an inherent limitation of retrospective studies, additional potentially important variables were not available from the prospective database. This concerns, for example, the amount of opioids given after right and left colectomy, respectively. However, large differences appear to be unlikely with exactly the same perioperative care pathway for both comparative groups. Finally, the study sample is modest and these results have to be confirmed by future studies.

In conclusion, the present study provides some evidence for slower gastrointestinal recovery after right colon resections as compared to left colectomies. Given the similar overall compliance of perioperative care and wellmatched groups, it can be hypothesized that this is mainly linked to the type of surgical procedure. The mechanism remains to be clarified. As a consequence, we have adapted our ERAS protocol by recommending half-size portion until bowel recovery and a careful monitoring of ileus symptoms after right colectomy.

\section{Compliance with ethical standards}

Disclosure No external funding was required for this study.

\section{References}

1. Greco M, Capretti G, Beretta L et al (2014) Enhanced recovery program in colorectal surgery: a meta-analysis of randomized 
controlled trials. World J Surg 38:1531-1541. doi:10.1007/ s00268-013-2416-8

2. Rawlinson A, Kang P, Evans J, Khanna A (2011) A systematic review of enhanced recovery protocols in colorectal surgery. Ann R Coll Surg Engl 93:583-588

3. Gustafsson UO, Hausel J, Thorell A et al (2011) Adherence to the enhanced recovery after surgery protocol and outcomes after colorectal cancer surgery. Arch Surg 146:571-577

4. Roulin D, Donadini A, Gander S et al (2013) Cost-effectiveness of the implementation of an enhanced recovery protocol for colorectal surgery. Br J Surg 100:1108-1114

5. Wind J, Polle SW, Fung Kon Jin PHP et al (2006) Systematic review of enhanced recovery programmes in colonic surgery. Br J Surg 93:800-809

6. Gustafsson UO, Scott MJ, Schwenk W et al (2013) Guidelines for perioperative care in elective colonic surgery: enhanced recovery after surgery (ERAS $\left.\left({ }^{\circledR}\right)\right)$ society recommendations. World J Surg 37:259-284. doi:10.1016/j.clnu.2012.08.013

7. Cakir H, van Stijn MFM, Lopes Cardozo AMF et al (2013) Adherence to enhanced recovery after surgery and length of stay after colonic resection. Colorectal Dis 15:1019-1025

8. Veyrie N, Ata T, Muscari F et al (2007) Anastomotic leakage after elective right versus left colectomy for cancer: prevalence and independent risk factors. J Am Coll Surg 205:785-793

9. Kwaan MR, Al-Refaie WB, Parsons HM et al (2013) Are rightsided colectomy outcomes different from left-sided colectomy outcomes?: study of patients with colon cancer in the ACS NSQIP database. JAMA Surg 148:504-510

10. Bakker IS, Grossmann I, Henneman D et al (2014) Risk factors for anastomotic leakage and leak-related mortality after colonic cancer surgery in a nationwide audit. Br J Surg 101:424-432 (discussion 432)

11. Hinojosa MW, Konyalian VR, Murrell ZA et al (2007) Outcomes of right and left colectomy at academic centers. Am Surg 73: 945-948

12. Benedix F, Kube R, Meyer F et al (2010) Comparison of 17,641 patients with right- and left-sided colon cancer: differences in epidemiology, perioperative course, histology, and survival. Dis Colon Rectum 53:57-64

13. Masoomi H, Buchberg B, Dang P et al (2011) Outcomes of right vs. left colectomy for colon cancer. J Gastrointest Surg 15:2023-2028

14. Rana AR, Cannon JA, Mostafa G et al (2007) Outcomes of rightcompared with left-side colectomy. Surg Innov 14:91-95

15. Senagore AJ, Duepree HJ, Delaney CP et al (2003) Results of a standardized technique and postoperative care plan for laparoscopic sigmoid colectomy: a 30-month experience. Dis Colon Rectum 46:503-509

16. Neutzling CB, Lustosa SA, Proenca IM et al (2012) Stapled versus handsewn methods for colorectal anastomosis surgery. Cochrane Database Syst, Rev

17. Hübner M, Larson DW, Wolff BG (2012) "How I do it"—radical right colectomy with side-to-side stapled ileo-colonic anastomosis. J Gastrointest Surg 16:1605-1609

18. Roulin D, Blanc C, Muradbegovic M et al (2014) Enhanced recovery pathway for urgent colectomy. World J Surg 38:21532159. doi:10.1007/s00268-014-2518-y

19. Vather R, Trivedi S, Bissett I (2013) Defining postoperative ileus: results of a systematic review and global survey. J Gastrointest Surg 17:962-972

20. Dindo D, Demartines N, Clavien P-A (2004) Classification of surgical complications: a new proposal with evaluation in a cohort of 6336 patients and results of a survey. Ann Surg 240:205-213
21. Moghadamyeghaneh Z, Hwang GS, Hanna MH et al (2015) Risk factors for prolonged ileus following colon surgery. Surg Endosc 30:603-609

22. Vather R, Bissett I (2013) Risk factors for the development of prolonged post-operative ileus following elective colorectal surgery. Int J Colorectal Dis 28:1385-1391

23. Xu C, Chi P (2014) Relevant factor analysis on postoperative ileus following radical resection for colorectal cancer. Zhonghua Wei Chang Wai Ke Za Zhi Chin J Gastrointest Surg 17:361-364

24. Xu C, Chi P (2012) Comparison of the incidence of postoperative ileus following laparoscopic and open radical resection for colorectal cancer: a meta-analysis. Zhonghua Wei Chang Wai Ke Za Zhi Chin J Gastrointest Surg 15:1044-1047

25. Chapuis PH, Bokey L, Keshava A et al (2013) Risk factors for prolonged ileus after resection of colorectal cancer: an observational study of 2400 consecutive patients. Ann Surg 257:909-915

26. Svatek RS, Fisher MB, Williams MB et al (2010) Age and body mass index are independent risk factors for the development of postoperative paralytic ileus after radical cystectomy. Urology 76:1419-1424

27. Vather R, Josephson R, Jaung R et al (2015) Development of a risk stratification system for the occurrence of prolonged postoperative ileus after colorectal surgery: a prospective risk factor analysis. Surgery 157:764-773

28. Wolthuis AM, Bislenghi G, Fieuws S et al (2016) Incidence of prolonged postoperative ileus after colorectal surgery: a systematic review and meta-analysis. Colorectal Dis 18:O1-O9

29. Pikarsky AJ, Saida Y, Yamaguchi T et al (2002) Is obesity a high-risk factor for laparoscopic colorectal surgery? Surg Endosc 16:855-858

30. Ay AA, Kutun S, Ulucanlar $H$ et al (2011) Risk factors for postoperative ileus. J Korean Surg Soc 81:242-249

31. Millan M, Biondo S, Fraccalvieri D et al (2012) Risk factors for prolonged postoperative ileus after colorectal cancer surgery. World J Surg 36:179-185. doi:10.1007/s00268-011-1339-5

32. Thoker M, Wani I, Parray FQ et al (2014) Role of diversion ileostomy in low rectal cancer: a randomized controlled trial. Int J Surg 12:945-951

33. ERAS Compliance Group (2015) The impact of enhanced recovery protocol compliance on elective colorectal cancer resection: results from an international registry. Ann Surg 261:1153-1159

34. Ramírez JM, Blasco JA, Roig JV et al (2011) Enhanced recovery in colorectal surgery: a multicentre study. BMC Surg 11:9

35. Nelson R, Edwards S, Tse B (2007) Prophylactic nasogastric decompression after abdominal surgery. Cochrane Database Syst Rev 3:CD004929

36. Postoperative Pain Forum Group, Marret E, Remy C, Bonnet F (2007) Meta-analysis of epidural analgesia versus parenteral opioid analgesia after colorectal surgery. Br J Surg 94:665-673

37. Levy BF, Scott MJ, Fawcett W et al (2011) Randomized clinical trial of epidural, spinal or patient-controlled analgesia for patients undergoing laparoscopic colorectal surgery. $\mathrm{Br}$ J Surg 98: 1068-1078

38. Hübner M, Blanc C, Roulin D et al (2014) Randomized clinical trial on epidural versus patient-controlled analgesia for laparoscopic colorectal surgery within an enhanced recovery pathway. Ann Surg 261:648-653

39. Bragg D, El-Sharkawy AM, Psaltis E et al (2015) Postoperative ileus: recent developments in pathophysiology and management. Clin Nutr 34:367-376

40. Simorov A, Thompson J, Oleynikov D (2014) Alvimopan reduces length of stay and costs in patients undergoing segmental colonic resections: results from multicenter national administrative database. Am J Surg 208:919-925 (discussion 925) 
41. Abodeely A, Schechter S, Klipfel A et al (2011) Does alvimopan enhance return of bowel function in laparoscopic right colectomy? Am Surg 77:1460-1462

42. Kelley SR, Wolff BG, Lovely JK, Larson DW (2013) Fast-track pathway for minimally invasive colorectal surgery with and without alvimopan (Entereg) ${ }^{\mathrm{TM}}$ : which is more cost-effective? Am Surg 79:630-633

43. Tan EK, Cornish J, Darzi AW, Tekkis PP (2007) Meta-analysis: alvimopan vs. placebo in the treatment of post-operative ileus. Aliment Pharmacol Ther 25:47-57

44. Sun Y, Li T, Wang N et al (2012) Perioperative systemic lidocaine for postoperative analgesia and recovery after abdominal surgery: a meta-analysis of randomized controlled trials. Dis Colon Rectum 55:1183-1194

45. Short V, Herbert G, Perry R et al (2015) Chewing gum for postoperative recovery of gastrointestinal function. Cochrane Database Syst Rev 2:CD006506

46. Müller SA, Rahbari NN, Schneider F et al (2012) Randomized clinical trial on the effect of coffee on postoperative ileus following elective colectomy. Br J Surg 99:1530-1538

47. Zingg U, Miskovic D, Pasternak I et al (2008) Effect of bisacodyl on postoperative bowel motility in elective colorectal surgery: a prospective, randomized trial. Int J Colorectal Dis 23:1175-1183 